$$
=\left(V U^{\prime} g_{a}, g_{b}\right)
$$

from the extension of (18). Since $b$ is arbitrary, it follows that $m(a, x)$ $=V U^{\prime} g_{a}$ almost everywhere. In a similar manner, starting from (8), we can also prove that $n(a, x)=V^{\prime} U g_{a}$ almost everywhere.

\title{
REFERENCES
}

1. S. Bochner, Inversion formulae and unitary transforms, Ann. of Math. vol. 55 (1934) pp. 111-115.

2. S. Bochner and K. Chandrasekharan, Fourier transforms, Princeton University Press.

McGill University

\section{A PROPERTY OF THE LAPLACE TRANSFORMATION}

P. G. ROONEY

1. Introduction. While certain of the properties of the Laplace transformation are so well known that they have become engineering tools, there are others that have received very little attention, and yet are very interesting. One of these comes about as follows. Let $f(s)$ be the Laplace transform of $\phi(t)$, that is

I

$$
f(s)=\int_{0}^{\infty} e^{-s t} \phi(t) d t=\mathfrak{L}(\phi(t) ; s) .
$$

Then under certain conditions,

II

$$
\mathcal{L}\left(\phi\left(t^{2}\right) ; s\right)=\frac{s}{4 \pi^{1 / 2}} \int_{0}^{\infty} e^{-y} y^{-3 / 2} f\left(\frac{s^{2}}{4 y}\right) d y ;
$$

this formula is given, for example, in $[2,4.1(22)]$. At least one generalization of this formula is known, that giving $\mathscr{L}\left(t^{\nu} \phi\left(t^{2}\right) ; s\right)$-see $[2,4.1(22)$ and $(23)]$ - but we propose to generalize here in a different direction, namely that of replacing the $y^{-3 / 2}$ in the right-hand integral of II by $y^{\nu-1}$. Specifically, we propose to show that, under certain conditions 1957.

Received by the editors November 29, 1956 and, in revised form, February 20, 
III

$$
\int_{0}^{\infty} t^{\nu+1} K_{\nu}(s t) \phi\left(t^{2}\right) d t=2^{\nu-2} s^{-\nu} \int_{0}^{\infty} e^{-y} y^{\nu-1} f\left(\frac{s^{2}}{4 y}\right) d y,
$$

where $K_{\nu}(x)$ is the modified Bessel function of the second kind. The theorem establishing formula III is given in section two.

The value of formula III lies in the fact that it converts an integral with a Bessel function kernel into one whose kernel is an elementary function, and thus gives some hope that the original integral may be evaluated. The fact that this conversion is performed via the medium of the Laplace transformation is even more fortunate since very extensive tables of Laplace transform pairs are in existence. In section three we shall evaluate several integrals by this method.

\section{Establishment of the formula.}

THEOREM. If either

(a) $\nu \geqq 0$, and $e^{-\gamma t^{1 / 2}} \phi(t) \in L(0, \infty)$ for some $\gamma>0$, or

(b) $\nu<0$, and $t^{\nu} e^{-\gamma t^{1 / 2}} \phi(t) \in L(0, \infty)$ for some $\gamma>0$, then $\mathfrak{L}(\phi(t) ; s)$ exists for all $s>0, \int_{0}^{\infty} t^{\nu+1} K_{\nu}(s t) \phi\left(t^{2}\right) d t$ exists for all $s>\gamma$, and for all $s>\gamma$, III holds.

PROOF FOR $\nu \geqq 0$. If $s>0$ and $t>0, e^{-\left(s t-\gamma t^{1 / 2}\right)} \leqq e^{\gamma^{2}} / 4 s=M_{1}$, so that $\int_{0}^{\infty} e^{-s t}|\phi(t)| d t \leqq M_{1} \int_{0}^{\infty} e^{-\gamma t^{1 / 2}}|\phi(t)| d t<\infty$, and $\mathscr{L}(\phi(t) ; s)$ exists.

For the existence of $\int_{0}^{\infty} t^{\nu+1} K_{\nu}(s t) \phi\left(t^{2}\right) d t$ for each $s>\gamma$, it suffices to show that for each $s>\gamma$

$$
I_{1}=\int_{0}^{\delta} t^{\nu+1}\left|K_{\nu}(s t) \phi\left(t^{2}\right)\right| d t, \quad \text { and } \quad I_{2}=\int_{R}^{\infty} t^{\nu+1}\left|K_{\nu}(s t) \phi\left(t^{2}\right)\right| d t
$$

are finite, for some $R>\delta>0$.

Consider first $I_{1}$. From $[1, \$ 7.2 .2(13)$ and (12)], it follows that $K_{\nu}(x)=O\left(x^{-\nu}\right)$ as $x \rightarrow 0+$. Hence if $s>0$ there is a constant $M_{2}$ such that $\left|K_{\nu}(x)\right| \leqq M_{2} x^{-\nu}$ for $0 \leqq x \leqq s \delta$. Thus

$$
\int_{0}^{\delta} t^{\nu+1}\left|K_{\nu}(s t) \phi\left(t^{2}\right)\right| d t \leqq M_{2} s^{-\nu} \int_{0}^{\delta} t\left|\phi\left(t^{2}\right)\right| d t<\infty,
$$

and hence $I_{1}$ is finite.

Now consider $I_{2}$. From $[1, \S 7.4 .1(1)], K_{\nu}(x) \sim(\pi / 2 x)^{1 / 2} e^{-x}$ as $x \rightarrow \infty$. Hence for each $s>\gamma$ there is a constant $M_{3}$ such that $\left|K_{\nu}(x)\right|$ $\leqq M_{3} x^{-1 / 2} e^{-x}$ for $x>s R$. Hence if $s>\gamma$,

$$
\int_{R}^{\infty} t^{\nu+1}\left|K_{\nu}(s t) \phi\left(t^{2}\right)\right| d t \leqq M_{3} s^{-1 / 2} \int_{R}^{\infty} t^{\nu+1 / 2} e^{-s t}\left|\phi\left(t^{2}\right)\right| d t .
$$

But since $s>\gamma, t^{\nu-1 / 2} e^{-(8-\gamma) t}$ is bounded for $t \geqq R$, say by $M_{4}$. Hence 
$t^{\gamma+1 / 2} e^{-8 t} \leqq M_{4} t e^{-\gamma t}$, and thus

$$
\begin{aligned}
\int_{R}^{\infty} t^{\nu+1}\left|K_{\nu}(s t) \phi\left(t^{2}\right)\right| d t & \leqq M_{3} M_{4} s^{-1 / 2} \int_{R}^{\infty} e^{-\gamma t} t\left|\phi\left(t^{2}\right)\right| d t \\
& \leqq M_{3} M_{4} s^{-1 / 2} \int_{0}^{\infty} e^{-\gamma t 1 / 2}|\phi(t)| d t<\infty .
\end{aligned}
$$

Hence $I_{2}$ is finite and

$$
\int_{0}^{\infty} t^{\nu+1} K_{\nu}(s t) \phi\left(t^{2}\right) d t \text { exists for } s>\gamma .
$$

But by $[2, \S 5.16(40)]$,

Hence

$$
K_{\nu}(x)=2^{\nu-1} x^{-\nu} \int_{0}^{\infty} e^{-\left(y+x^{2} / 4 y\right)} y^{\nu-1} d y .
$$

$$
\begin{aligned}
\int_{0}^{\infty} K_{\nu}(s t) t^{\nu+1} \phi\left(t^{2}\right) d t & =2^{\nu-1} s^{-\nu} \int_{0}^{\infty} t \phi\left(t^{2}\right) d t \int_{0}^{\infty} e^{-\left(y+s^{2} t^{2} / 4 y\right)} y^{\nu-1} d y \\
& =2^{\nu-1} s^{-\nu} \int_{0}^{\infty} e^{-y} y^{\nu-1} d y \int_{0}^{\infty} e^{-s^{2} t^{2} / 4 y} t \phi\left(t^{2}\right) d t \\
& =2^{\nu-2} s^{-\nu} \int_{0}^{\infty} e^{-y} y^{\nu-1} d y \int_{0}^{\infty} e^{-s^{2} u / 4 y} \phi(u) d u \\
& \left.=2^{\nu-2} s^{-\nu} \int_{0}^{\infty} e^{-y} y^{\nu-1} f\left(\frac{s^{2}}{4 y}\right) d y, \quad \text { (where } u=t^{2}\right)
\end{aligned}
$$

the interchange of integrations being valid for $s>\boldsymbol{\gamma}$ by Fubini's theorem.

ProOF FOR $\nu<0$. The existence of $\mathscr{L}(\phi(t) ; s)$ follows as in the previous case. The existence of $\int_{0}^{\infty} t^{\nu+1} K_{\nu}(s t) \phi\left(t^{2}\right) d t$ follows from proof (a) since $K_{-\nu}(x)=K_{\nu}(x), x>0$, and the proof of formula III follows then as in the previous case.

3. Applications. We give below three examples of the use of formula III, ranging upwards in complexity. Others, more complicated, can easily be found, but these three illustrate the method amply. Each of the integrals has, of course, been evaluated before.

ExAmpLE 1. Let $\phi(t)=t^{\mu-1}, \mu>0$. Then the theorem says in this case III is valid if $\mu+\nu>0$ and $s>0$. From [2, 4.3(1)], $f(s)=\Gamma(\mu) / s^{\mu}$, and III yields 


$$
\begin{aligned}
\int_{0}^{\infty} t^{2 \mu+\nu-1} K_{\nu}(s t) d t & =2^{2 \mu+\nu-2} s^{-(2 \mu+\nu)} \Gamma(\mu) \int_{0}^{\infty} e^{-y} y^{\mu+\nu-1} d y \\
& =2^{2 \mu+\nu-2} s^{-(2 \mu+\nu)} \Gamma(\mu) \Gamma(\mu+\nu),
\end{aligned}
$$

or changing st to $t$,

$$
\int_{0}^{\infty} t^{2 \mu+\nu-1} K_{\nu}(t) d t=2^{2 \mu+\nu-2} \Gamma(\mu) \Gamma(\mu+\nu) .
$$

EXAMPLE 2. $\phi(t)=t^{\mu / 2} J_{\mu}(a t)^{1 / 2}, a>0$. Then from the theorem, III is valid if $\mu>-1, \mu+\nu>-1, s>0$. From $[2,4.14(30)]$,

$$
\begin{aligned}
\int_{0}^{\infty} K_{\nu}(s t) J_{\mu}(a t) t^{\mu+\nu+1} d t & =2^{\mu+\nu} s^{-(2 \mu+\nu+2)} a^{\mu} \int_{0}^{\infty} e^{-\left(1+a^{2} / s^{2}\right) y} y^{\mu+\nu} d y \\
& =\frac{(2 a)^{\mu}(2 s)^{\nu} \Gamma(\mu+\nu+1)}{\left(a^{2}+s^{2}\right)^{\mu+\nu+1}} .
\end{aligned}
$$

EXAmple 3. $\phi(t)=J_{\mu}\left(a t^{1 / 2}\right) J_{\mu}\left(b t^{1 / 2}\right), a>0, b>0$. Then the theorem gives that III is valid for $\mu>-1, \mu+\nu>-1, s>0$. From $[1,7.7 .3(25)]$ $f(s)=s^{-1} e^{-\left(a^{2}+b^{2}\right) / 4 s} I_{\mu}(a b / 2 s)$, and III yields

$$
\int_{0}^{\infty} t^{\nu+1} K_{\nu}(s t) J_{\mu}(a t) J_{\mu}(b t) d t=2^{\nu} s^{-(\nu+2)} \int_{0}^{\infty} e^{-\left(a^{2}+b^{2}+s^{2}\right) y / s^{2}} y^{\nu} I_{\mu}\left(\frac{2 a b}{s^{2}} y\right) d y .
$$

This last integral can be evaluated by expanding $I_{\mu}(x)$ in its power series thus giving

$$
\begin{array}{r}
\int_{0}^{\infty} t^{\nu+1} K_{\nu}(s t) J_{\mu}(a t) J_{\mu}(b t) d t=\frac{(a b)^{\mu}(2 s)^{\nu}}{\left(a^{2}+b^{2}+s^{2}\right)^{\mu+\nu+1}} \frac{\Gamma(\mu+\nu+1)}{\Gamma(\nu+1)} \\
\cdot{ }_{2} F_{1}\left(\frac{\mu+\nu}{2}+1, \frac{\mu+\nu+1}{2} ; \nu+1 ; \frac{4 a^{2} b^{2}}{\left(a^{2}+b^{2}+s^{2}\right)^{2}}\right) .
\end{array}
$$

\section{REFERENCES}

1. A. Erdélyi et al., Higher transcendental functions II, New York, 1953.

2. - - Tables of integral transforms I, New York, 1954.

UNIVERSITY OF TORONTO 